\title{
Laser beam quality and pointing measurement with an optical resonator
}

\author{
Patrick Kwee, Frank Seifert, Benno Willke, and Karsten Danzmann \\ Max-Planck-Institut für Gravitationsphysik (Albert-Einstein-Institut), 30167 Hannover, Germany
}

(Received 21 January 2007; accepted 11 June 2007; published online 12 July 2007)

\begin{abstract}
We present a compact diagnostic breadboard that is based on an optical ring resonator for measuring beam quality and pointing of single-frequency continuous wave lasers at a wavelength of $1064 \mathrm{~nm}$. To determine the beam quality of the coherent test beam, this optical resonator is used to perform a mode decomposition into Hermite-Gaussian modes. For our laser system, a power fraction in the fundamental Gaussian mode of $97.2 \% \pm 0.2 \%$ was measured. Residual misalignment and mis-mode-matching to the resonator as well as the astigmatism and/or ellipticity of the test beam have been determined. Numerical simulations showed that measurements of the $M^{2}$ factor and transversal intensity distribution are not suitable for determining this power fraction. To measure the beam pointing, the fundamental mode of the optical resonator was used as a stable reference. The pointing of the test beam was measured with the differential wave front sensing technique up to Fourier frequencies of $1 \mathrm{kHz}$ with a sensitivity to relative pointing of $|\epsilon|=1 \times 10^{-6} / \sqrt{\mathrm{Hz}}$. Pointing measurements with an alternative method were performed and showed good agreement. (c) 2007 American Institute of Physics. [DOI: 10.1063/1.2754400]
\end{abstract}

\section{INTRODUCTION}

Many optical experiments need light sources of high beam quality. However, it is very difficult to define a general criterion for the beam quality. Often the deviation of the test beam from an ideal Gaussian fundamental mode $\left(\mathrm{TEM}_{00}\right)$ is used to characterize the beam quality. Different definitions have been established for the various fields of application in order to quantify the beam quality. If, for example, the beam propagation is important for the application, then the $M^{2}$ factor ${ }^{1}$ is often used. This factor primarily compares the divergence of the test beam with the divergence of an ideal $\mathrm{TEM}_{00}$ beam. In this field of applications, measurement methods have been established that accordingly measure the beam propagation. ${ }^{2}$

In other fields of applications, the power in the fundamental mode $\mathrm{TEM}_{00}$ compared with the overall power is a better criterion for the beam quality. This ratio will be called the fundamental power in this article. That fundamental power directly specifies the power fraction that can be coupled into an ideal optical resonator that is resonant to the $\mathrm{TEM}_{00}$ mode. This quantity can therefore be determined by measuring the coupling efficiency of the laser beam to a resonator. ${ }^{3,4}$

The fundamental power can also be determined by decomposing the beam into Hermite-Gaussian modes. The advantage of such a modal analysis is that significantly more information about the beam is gathered. There are several ideas and methods for a modal analysis of partially coherent beams. ${ }^{5-9}$ For fully coherent beams, which are considered here, other methods were developed that use spatial filters ${ }^{10}$ or the measurement of the transverse intensity distribution along the beam axis. ${ }^{11}$

We have developed a so-called diagnostic breadboard to characterize the beam quality of single-frequency continuous wave $(\mathrm{cw})$ lasers by performing a high resolution modal analysis of the fully coherent test beam.

The measurement method, which we call Modescan, decomposes the test beam with the aid of an optical ring resonator into an expansion of Hermite-Gaussian modes (with the exception of certain degeneracies). A program automatically analyzes the measurement and determines the fundamental power, among other parameters. Similar experiments are already described in the literature. However, only the fundamental power was determined in a manual evaluation of the measurement data ${ }^{3,4}$ or the data were used to optimize the alignment and the mode matching of the resonator. ${ }^{12}$

The sensitivity of the Modescan was experimentally investigated. In our case a fundamental power of $97.2 \%$ with an uncertainty of $\pm 0.2 \%$ of the laser beam used had been measured. Higher-order modes with relative powers of down to $2 \times 10^{-4}$ were detected.

An important application area for lasers is the interferometric gravitational wave detection. Since in these experiments the laser light is coupled into several optical resonators, the fundamental power is the appropriate quantity for measuring the beam quality. We have analyzed the feasibility of determining the fundamental power from $M^{2}$ measurements and from single transverse intensity measurements. Numerical simulations showed that only the Modescan among the methods analyzed can be used to measure the fundamental power with the desired accuracy.

Besides the beam quality, the pointing of the laser beam is of interest in a number of experiments. Pointing of a laser beam is described by a transverse shift as well as an angular tilt of the beam axis with respect to a reference axis at a specified position along the beam axis. Usually, position sensitive detectors (PSDs) are used to measure the transverse shift of the beam at one position along the beam axis. ${ }^{13-16}$ 
The angular tilt has to be transformed by a lens into a transverse shift in order to be measured. This transverse shift can then be detected with a second PSD at the focal plane of the lens.

The reference for this kind of pointing measurement is given by the positions of the PSDs. Since at least two PSDs are required to measure shift and tilt, a relative movement of the PSD with respect to each other or even movements of the optical components after the splitting point of the beam will lead to measurement errors.

We use the above-mentioned diagnostic breadboard to measure the pointing of the beam as well. Compared with other methods, our measurement method uses a real laser beam as the reference and measures the shift and tilt between the test beam and the reference beam by analyzing the interference of the test and the reference beam. The reference beam is the fundamental mode of a quasimonolithic ring resonator, which is excited by the test beam itself. To measure the relative shift and tilt, we use the differential wave front sensing (DWS) method. ${ }^{17}$ As an interferometric measurement method, it allows a very accurate measurement of relative shift and tilt of the beams.

Similar methods were already used to automatically optimize the alignment of a laser beam with respect to a resonator. In these experiments phase modulation techniques ${ }^{18,19}$ different from ours or even multiple phase modulations were used. $^{20}$ The aim of those experiments was to stabilize the laser beam to suspended resonators or to improve the frequency stability of the resonator by an active pointing suppression.

The measurement method and the experiment presented here were developed to measure the pointing of laser systems for interferometric gravitational wave detectors. Strict requirements ${ }^{21}$ for the allowed pointing of the laser system are set in this application area in order to reach the design sensitivity of the detectors.

The pointing of a single-frequency $\mathrm{cw}$ laser system with an output wavelength of $1064 \mathrm{~nm}$ is presented up to a Fourier frequency of $1 \mathrm{kHz}$. Measurements with an alternative method were performed with good agreement. A sensitivity to relative pointing of $|\epsilon|=1 \times 10^{-6} / \sqrt{\mathrm{Hz}}$ was achieved [for definition of $\epsilon$, see Eq. (19)]. Since transverse shift and angular tilt were measured simultaneously, other parameters that are used to quantify beam pointing can be calculated.

With the compact diagnostic unit presented in this article, it will be possible to optimize the fundamental power of lasers by performing adjustments, to monitor the short and long term performance of laser systems, and to further investigate the causes of pointing fluctuations in order to possibly reduce them.

\section{MODESCAN}

Every laser beam in the paraxial approximation can be expanded into a series of Hermite-Gaussian modes. ${ }^{22}$ They form an orthogonal complete set of functions. Therefore, the complex amplitude $U(\mathbf{r})$ of a single-frequency laser beam can be expressed as

$$
U(\mathbf{r})=\sum_{l, m \geqslant 0} c_{l, m} \Phi_{l, m}(\mathbf{r}),
$$

where $\Phi_{l, m}$ is the complex amplitude of the $\mathrm{TEM}_{l m}$ mode and $c_{l, m}$ are the complex expansion coefficients, which are also called modal weights. With this representation, the fundamental power $P_{\text {fund }} / P_{\text {tot }}$ is given by

$$
P_{\text {fund }} / P_{\text {tot }}=\frac{\left|c_{0,0}\right|^{2}}{P_{\text {tot }}} \quad \text { with } P_{\text {tot }}=\sum_{l, m}\left|c_{l, m}\right|^{2} \text {. }
$$

With the aid of an optical resonator, this expansion into $\mathrm{TEM}_{l m}$ modes can be partly measured because the resonance frequencies of $\mathrm{TEM}_{l m}$ resonator eigenmodes are generally different. The resonance frequencies of modes with the same order $g=l+m$ are, however, degenerated in a linear resonator. The power $p_{g}$ of the modes with order $g$ is given by

$$
p_{g}=\sum_{l=0}^{g}\left|c_{l, g-l}\right|^{2} .
$$

With this definition, the power buildup in a resonator can be calculated dependent on the round trip length $L$ of the resonator. It is expressed by a superposition of terms with Airy denominators,

$$
P(L) \propto \sum_{g} \frac{p_{g}}{1+(2 \mathcal{F} / \pi)^{2} \sin ^{2}\left(-\pi L / \lambda+\zeta_{0}(1+g) / 2\right)},
$$

where $\mathcal{F}$ is the finesse of the resonator, $\lambda$ the wavelength of the laser, and $\zeta_{0}$ the Gouy phase of one round trip in the resonator. $^{23}$

The idea of our measurement method is to couple the test beam into a resonator and to measure the transmitted power of the resonator with a photodiode dependent on the length $L$. Afterward, $P(L)$ is fitted in a multistage procedure to the measurement. Thus, one receives the parameters $p_{g}$, which can be used to easily calculate the fundamental power,

$$
P_{\text {fund }} / P_{\text {tot }}=\frac{p_{0}}{P_{\text {tot }}} .
$$

Besides this quantity, significantly more information about the modal composition of the beam can be gathered compared with measurement methods that determine only the fundamental power. So it is possible, as will be described later on [see Eq. (11)], to determine the astigmatism and ellipticity of the beam or to precisely calculate the spatial filter effect of a resonator concerning the overall highermode power.

This measurement method works best for singlefrequency lasers, since then no distinction between different longitudinal modes is needed. The frequency of the laser has to be sufficiently stable, so that an identification of the modes by their resonance frequency is possible.

The influence of intensity noise can be estimated assuming that the relative intensity noise of the beam that is coupled into the resonator and that is transmitted by the resonator are equal (this is not generally true but a valid assumption for noise frequencies much smaller than the resonator 


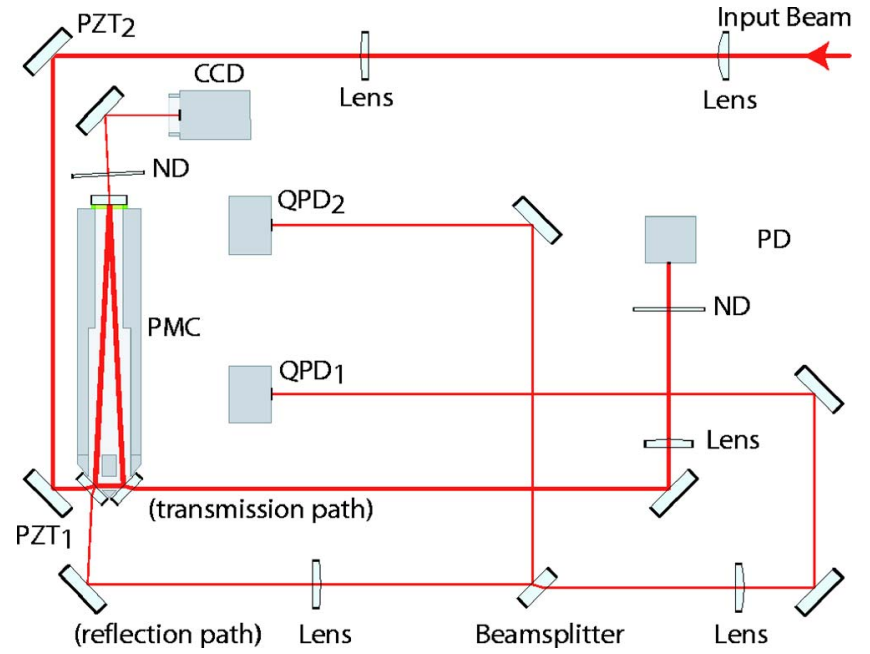

FIG. 1. (Color online) Schematic overview of the experimental setup. Details of the purpose of the components are given in the text. $\mathrm{PMC}=$ pre-mode cleaner $=$ optical resonator; $\mathrm{QPD}=$ quadrant photodiode; $\mathrm{PD}=$ photodiode; $\mathrm{ND}=$ neutral density filter; $\mathrm{CCD}=\mathrm{CCD}$ camera; $\mathrm{PZT}=$ mirror with piezoelectric elements.

bandwidth). Using the following approximation for the power fraction of higher modes, the error by intensity noise can be roughly estimated:

$$
1-P_{\text {fund }} / P_{\text {tot }} \approx \sum_{g>0} p_{g} / p_{0} .
$$

Thus, the relative error of this power fraction is about twice as high as the relative intensity noise of the beam in the measurement bandwidth, assuming the worst case where the sums over the $p_{g}$ 's and $p_{0}$ have the same relative error as the power of the beam.

In principle, it is also possible to determine the modal weights by measuring the power reflected by the resonator. However, this has the disadvantage that the error of the modal weights is proportional to the absolute intensity noise and is thus large for very weak modes.

\section{A. Setup}

The experiment was set up on a transportable breadboard $\left(60 \times 45 \mathrm{~cm}^{2}\right)$ (Fig. 1). A ring resonator similar to Refs. 3 and 24 with two plane $45^{\circ}$ mirrors, 1 in. wide, with a power transmission of $1.5 \%$ (all quantities are given for $p$-polarized light) and one curved high-reflectivity mirror with a curvature of $R=-1 \mathrm{~m}$ was used. The round trip length is $L=420 \mathrm{~mm}$, which can be changed by about $1.3 \mu \mathrm{m}$ with a piezoelectric element at the curved mirror. The free spectral range (FSR) is $715 \mathrm{MHz}$. The finesse was measured as $\mathcal{F} \approx 200$ at a wavelength of $1064 \mathrm{~nm}$. The waist radius of the fundamental mode is $w_{0}=372 \mu \mathrm{m}$ with a half divergence angle of $\theta_{D}=910 \mu \mathrm{rad}$. The Gouy phase of the fundamental mode for one round trip is $\zeta_{0}=0.95 \mathrm{rad}(=0.15 \mathrm{FSR})$.

Since the phase shift and the reflectivity of the resonator mirror coating depends on the polarization of the light, the resonance frequencies are different for $s$ - and $p$-polarized light. Therefore, the test beam should be $p$-polarized with ratios of $1: 100$ to $1: 1000$ to avoid misinterpretations. The resonator is very close to being impedance matched, since the input and the output coupler mirrors were coated in the same coating run.

The eigenmodes of the resonator were in good approximation to the Hermite-Gaussian modes, because the smallest aperture was about ten times larger than the beam width at this point. Since the beam in the resonator had an incidence angle of about $3^{\circ}$ at the curved mirror, the effective radius of curvature is different for the horizontal and vertical directions. This leads to small differences of $\delta w_{0} \approx 0.3 \mu \mathrm{m}$ between the horizontal and vertical beam waist radii. The eigenmodes of the resonator therefore have a small ellipticity. This further causes a small difference in the Gouy phase for $\mathrm{TEM}_{l 0}$ and $\mathrm{TEM}_{0 m}$ modes that in turn causes a splitting of the resonance frequencies for modes of the same order. This splitting cannot, however, be resolved with the low finesse of 200. Since the test beam is coupled into the resonator by a $45^{\circ}$ mirror, an astigmatism of $\delta z=1.6 \mathrm{~mm}$ is added to the test beam, caused by transmission through the substrate of $d=6.35 \mathrm{~mm}$ thickness.

The transmitted beam of the resonator is focused with a lens onto an InGaAs photodiode with an active area diameter of $1 \mathrm{~mm}$. The fundamental mode beam has a radius of $100 \mu \mathrm{m}$ at the photodiode. A transimpedance amplifier was used to read out the photodiode, which had a bandwidth of several megahertz, so that the sharp peaks due to the Airy denominators were completely resolved for the scanning frequency chosen (see Sec. II B). The maximum power on the photodiode was $15 \mathrm{~mW}$, which was within the linear range of the detector.

\section{B. Measurement and analysis}

The measurement was performed as follows. First of all, the test beam was aligned and mode matched to the resonator. Two lenses in front of the resonator were used to match the waist radius and position of the test beam to the waist of the resonator. The alignment of the test beam was adjusted with two mirrors, which could be tilted manually as well as electronically by piezoelectric elements. The alignment was automatically optimized by feedback loops, so that the degree of freedom was reduced from six (four times alignment, two times mode matching) to two manual degrees. This led to an acceleration of the mode-matching procedure. The sensing technique for the alignment of the test beam will be described later in Sec. III. A good alignment and mode matching is crucial for this measurement method, since higher $\mathrm{TEM}_{l m}$ modes are excited by a misalignment or mismode-matching and will therefore reduce the fundamental power determined. The quality of the alignment and mode matching can, however, be determined from the measurement data, as will be described later in this section.

In the next step, a high voltage ramp signal at $12 \mathrm{~Hz}$ was applied to the piezo of the resonator. Thus, a microscopic length change of the resonator caused a periodic shift of the resonance frequencies of about 2.5 FSR. The transmitted power was sampled together with the ramp signal at the piezo for one FSR. According to this the measurement duration was about $17 \mathrm{~ms}$. About 4000 data points were sampled per FSR. The sampling rate was about $240 \mathrm{ksamples} / \mathrm{s}$ which is 
slower than the storage time of the resonator, which is about $90 \mathrm{~ns}$. Hence, a stationary state in the resonator can be assumed, which is the precondition for Eq. (4). The frequency fluctuations of the test beam should not exceed a root-meansquare (rms) value of about $350 \mathrm{kHz}$ in a Fourier frequency band from $59 \mathrm{~Hz}$ up to $240 \mathrm{kHz}$ in order to determine the resonance frequencies of the modes within $1 / 10$ of the linewidth of the resonator.

Since the expansion of the piezo at the resonator depended nonlinearly on the applied voltage and showed a hysteresis, a calibration of the piezo was necessary. After the calibration, we were able to calculate the length change of the resonator from the sampled ramp signal.

The model $P(L)$ given by Eq. (4) was now fitted to the measurement in order to determine the parameters $p_{g}$. The measured data was filtered by a convolution with a Gauss function to reduce measurement noise. Then the positions of the resonance peaks were determined.

Next, the order of the modes for each peak was assigned. If only the fundamental power is to be determined, this step can be skipped. The determined peaks were assigned to the closest TEM $_{l m}$ mode with $l+m$ not exceeding a predefined upper limit. Twice as many modes were considered as peaks were found in the measurement. The theoretical positions of the modes were calculated, where one has to consider that the resonance frequencies of the $\mathrm{TEM}_{l m}$ modes did not only depend on the order $g=l+m$ but also on whether $l$ is even or odd for the resonator used. The field distribution is mirrored at the vertical axis at each mirror. For modes with an asymmetric field distribution to the vertical axis (modes with odd l), this corresponds to an additional phase shift of $180^{\circ}$ for three reflections per round trip.

After this identification of the peaks based on their resonance frequency, the parameters $p_{g}$ were calculated. A system of linear equations was solved, such that the model $P(L)$ matched the peaks of the actual measurement. This fit procedure was automated by a program and solely one parameter for the filtering has to be adjusted manually depending on the measurement noise.

If the test beam has a high fraction of its power in the $\mathrm{TEM}_{00}$ mode, then the excitation of $\mathrm{TEM}_{10}$ and $\mathrm{TEM}_{01}$ modes in the resonator can be reduced arbitrarily by alignment. If the test beam is transverse shifted by $\delta x$ and angular tilted by $\delta \alpha$ in the $x$ or $y$ direction with respect to the fundamental mode of the resonator, then the power of the $\mathrm{TEM}_{10}$ or, respectively, $\mathrm{TEM}_{01}$ mode can be calculated to first order. ${ }^{25}$ From the power in the $\mathrm{TEM}_{10}$ and $\mathrm{TEM}_{01}$ modes, the quality of the alignment of the test beam can be determined conversely,

$$
\begin{aligned}
& \delta x=w_{0} \sqrt{\frac{p_{1}}{P_{\text {tot }}}} \sin (\phi), \\
& \delta \alpha=\theta_{D} \sqrt{\frac{p_{1}}{P_{\text {tot }}}} \cos (\phi) .
\end{aligned}
$$

The angle $\phi$ cannot be determined from the Modescan measurement, since the phases of $c_{1,0}$ and $c_{0,1}$ cannot be measured with this method.
Using this principle, the quality of the mode matching can be determined as well, by investigating the power $p_{2}$ of the modes with order 2 and even $l$. These are the $\mathrm{TEM}_{20}$ and the $\mathrm{TEM}_{02}$ modes. They indicate a mismatch of the waist radius or the waist size.

Since two spherical lenses instead of four cylinder lenses were used for the mode matching, the waist radius and position cannot be altered independently for the $x$ and $y$ directions. Therefore, it is in general not possible to reduce the excitation of theses modes arbitrarily. By expressing the subspace that is spanned by the $\mathrm{TEM}_{20}$ and $\mathrm{TEM}_{02}$ modes in another basis using the Laguerre-Gaussian modes, ${ }^{26}$ the modes that can and that cannot be altered by the two spherical lenses can be separated,

$$
\Phi_{2,0}=\frac{1}{\sqrt{2}}\left(\Lambda_{0,2}-\Lambda_{1,0}\right), \quad \Phi_{0,2}=-\frac{1}{\sqrt{2}}\left(\Lambda_{0,2}+\Lambda_{1,0}\right),
$$

where $\Lambda_{p, l}$ is the complex amplitude of the LaguerreGaussian mode $\operatorname{Lag}_{p l}$. The power fraction of the $\operatorname{Lag}_{10}$ mode can be reduced arbitrarily by optimizing the lens positions. The power of the $\mathrm{Lag}_{02}$ mode originates, however, from an astigmatism and/or an ellipticity of the test beam,

$$
\left.\begin{array}{c}
\delta w_{0}=w_{0} \sqrt{\frac{p_{2}}{P_{\text {tot }}}} \sin (\phi) \sin (\kappa) \\
\delta z=z_{0} \sqrt{\frac{p_{2}}{P_{\text {tot }}}} \cos (\phi) \sin (\kappa)
\end{array}\right\} \quad \operatorname{Lag}_{10} \text { fraction, (10) }
$$

where $z_{x}$ and $z_{y}$ are the positions of the waist in the $x$ and $y$ directions. The three angles $\phi, \tau$, and $\kappa$ cannot be determined from the measurement, since the phases of $c_{2,0}$ and $c_{0,2}$ as well as the power ratio $\left|c_{2,0}\right|^{2} /\left|c_{0,2}\right|^{2}$ cannot be measured with the Modescan. With an additional measurement of the spatial intensity distribution, the angle $\kappa$ can be calculated, as will be explained later (Sec. II D).

The fundamental mode of the resonator also has, as already mentioned, an ellipticity and an astigmatism outside of the resonator. This corresponds in first order to $\mathrm{Lag}_{02}$ mode with a relative power of $6 \times 10^{-5}$. This deviation from an ideal $\mathrm{TEM}_{00}$ mode can be neglected since it is almost below the detection limit. A mode can only be detected if the mode power is larger than the background created by all other modes. The background of the $\mathrm{TEM}_{00}$ mode at the position of the $\operatorname{Lag}_{02}$ mode is $9 \times 10^{-5}$.

\section{Alternative measurement methods}

For our experiments in the area of the interferometric detection of gravitational waves, the fundamental power is the crucial quantity to characterize the beam quality. We have compared the Modescan with two alternative measure- 
ment methods conventionally used to measure the beam quality in the aspect of determining the fundamental power of a beam.

A fast and easily performed measurement method is the measurement of the transverse intensity distribution of the beam at one position along the beam axis using a chargecoupled device (CCD) camera. With this measured intensity distribution $I(x, y)$, the following quantity $H$ can be calculated:

$$
H=1-\frac{\int d x d y\left|I(x, y)-I_{\mathrm{fit}}(x, y)\right|}{\int d x d y I_{\mathrm{fit}}(x, y)},
$$

where $I_{\text {fit }}(x, y)$ is a fit of a Gaussian distribution to the measured data $I(x, y)$ with the constraint that the power and centroid of the two distributions are identical. The quantity $H$ is calculated by the readout software of our CCD camera and is called Gaussian fit. ${ }^{27}$ The correlation between the fundamental power of a beam and $H$ was determined in a numerical simulation. To simplify matters, the simulation was performed on unidimensional beams. The following complex amplitudes $U_{t}$ were used as test beams:

$$
U_{t}=\sqrt{\gamma} \Phi_{0,0}+\sqrt{1-\gamma} e^{i \varphi} \times\left\{\begin{array}{l}
\Phi_{3,0} \\
\Phi_{4,0} \\
\Phi_{5,0} \\
(1 / \sqrt{2})\left(\Phi_{3,0}+\Phi_{5,0}\right) .
\end{array}\right.
$$

As can be seen from the definition, the fundamental power of these beams is $\gamma$. The phase $\varphi$ between the $\mathrm{TEM}_{00}$ mode and the higher modes was altered in the simulation.

Another common measurement method is the determination of the $M^{2}$ factor. The correlation between the fundamental power and the $M^{2}$ factor was calculated for the beams just mentioned, using the definition of the $M^{2}$ factor given in Ref. 1 (since coherent beams are investigated, the simple formula given in Ref. 1 cannot be used to calculate the $M^{2}$ factor from the modal weights).

For comparison the $M^{2}$ factor of the real test beam was measured with a CCD camera. Therefore, the beam width was measured at 31 equidistant positions up- and downstream of the beam waist. The second moment of the transverse intensity distribution was used to calculate the beam width. A fit to the measured beam propagation was then used to calculate the cylindrical $M^{2}$ factor.

\section{Results}

The Modescan was used to measure the fundamental power of several laser systems. Among them were Nd:YAG (yttrium aluminum garnet) solid state ring lasers, $\mathrm{Nd}: \mathrm{YVO}_{4}$ solid state amplifiers, and ytterbium doped photonic crystal fiber amplifiers. ${ }^{28}$

The measurements presented here were performed with a three-head $\mathrm{Nd}: \mathrm{YVO}_{4}$ amplifier. The amplifier was a prototype system which was seeded by a ring laser ${ }^{29}$ with $12 \mathrm{~W}$ output power. The output power of the amplifier was $35 \mathrm{~W}$ at $1064 \mathrm{~nm} .120 \mathrm{~mW}$ was used from the main beam to measure

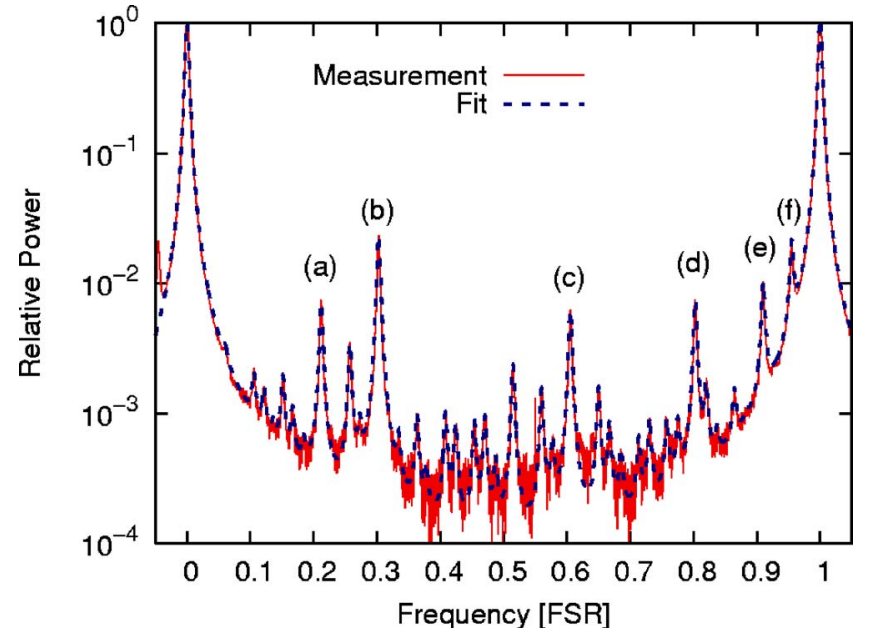

FIG. 2. (Color online) Modescan of a test beam. The measurement and fit of $P(L)$ are shown. Further details of the marked modes are given in Figs. 3 and 4.

the beam quality. The rms frequency noise of the laser was about $1.3 \mathrm{kHz}$ in the measurement frequency band. This is far below the estimated requirement of $350 \mathrm{kHz}$.

The fit program identified 37 higher transversal modes in the Modescan of the laser (see Figs. 2 and 3). A fundamental power of $91.5 \%$ was calculated and the fit to the data is very good in a qualitative way. Higher modes with relative powers ranging from $2.3 \times 10^{-2}$ down to $1.6 \times 10^{-4}$ were detected.

With a power of $0.14 \%$, each of the $\mathrm{TEM}_{10}$ and $\mathrm{TEM}_{01}$ modes contributes to the $8.5 \%$ of the overall power in higher modes (Fig. 3). This corresponds to the following deviations from the optimal alignment of the test beam in the $x$ as well as in the $y$ direction:

$$
\begin{aligned}
& \delta x=14 \mu \mathrm{m} \times \sin (\phi), \\
& \delta \alpha=34 \mu \mathrm{rad} \times \cos (\phi) .
\end{aligned}
$$

The power of the modes with order 2 and even $l$ is $2.3 \%$ of the total beam power (Fig. 3, label b). This correlates to the following deviation from the optimal mode matching and/or the following ellipticity and astigmatism, respectively:

$$
\left.\begin{array}{l}
\delta w_{0}=57 \mu \mathrm{m} \times \sin (\phi) \sin (\kappa) \\
\delta z=124 \mathrm{~mm} \times \cos (\phi) \sin (\kappa)
\end{array}\right\} \quad \operatorname{Lag}_{10} \text { fraction, }
$$

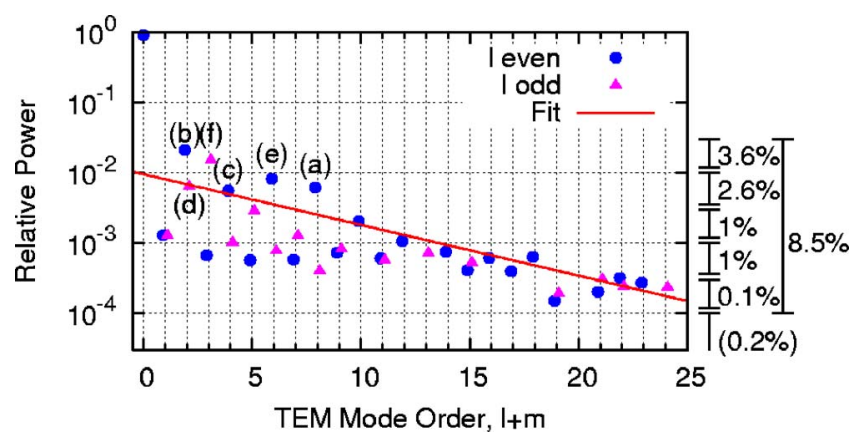

FIG. 3. (Color online) Relative power of the identified higher modes. A fit was calculated to estimate the power of unidentified modes with order greater than 24 . At the right border the sum powers in the indicated intervals are given. 


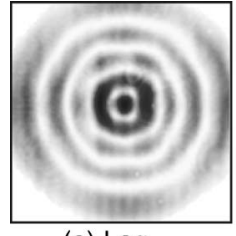

(a) $\operatorname{Lag}_{4,0}$

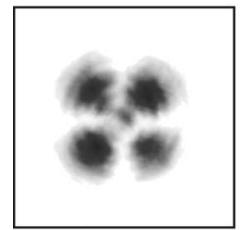

(d) TEM $_{1,1}$

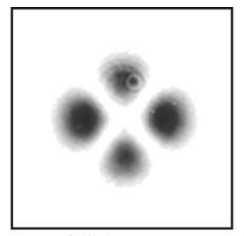

(b) $\operatorname{Lag}_{0,2}$

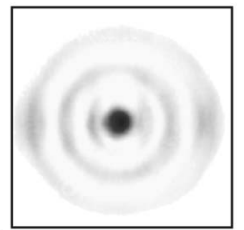

(e) $\operatorname{Lag}_{3,0}$ and $\mathrm{TEM}_{6,0}$

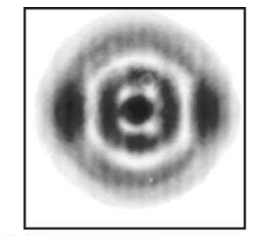

(c) $\operatorname{Lag}_{2,0}$ and TEM $_{4,0}$

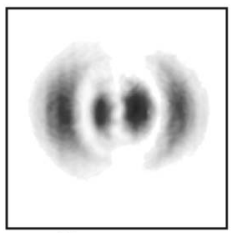

(f) $\mathrm{TEM}_{3,0}$
FIG. 4. Measured transversal intensity distributions of the six most powerful higher modes from Figs. 2 and 3. The scale is arbitrary for each distribution.

$$
\left.\begin{array}{c}
w_{0 x}-w_{0 y}=29 \mu \mathrm{m} \times \sin (\tau) \cos (\kappa) \\
z_{x}-z_{y}=62 \mathrm{~mm} \times \cos (\tau) \cos (\kappa)
\end{array}\right\} \quad \operatorname{Lag}_{02} \text { fraction }
$$

From the observed spatial intensity distribution of these modes (see Fig. 4, label b), one can recognize that most of the power is caused by the $\operatorname{Lag}_{02}$ mode. This corresponds to a small value of $\kappa$. The intensity in the center of the distribution is proportional to the $\operatorname{Lag}_{10}$ mode power since the field amplitude of the $\mathrm{Lag}_{02}$ vanishes at this point. The center of the measured intensity distribution was below the detection limit of the CCD camera. Therefore, the power of the $\operatorname{Lag}_{10}$ was below the detection limit and the mode matching was good compared with the astigmatism and the ellipticity of the beam. (The astigmatism and ellipticity of the test beam could have been compensated by using cylindrical lenses.)

Several experiments were performed in order to estimate the precision of the Modescan. The identification of the modes by their resonance frequency was checked by measuring the transversal intensity distribution of the six most powerful modes in the transmission of the resonator with a CCD camera. In each case the corresponding $\mathrm{TEM}_{l m}$ mode order was determined correctly (see Figs. 3 and 4).

To estimate the statistical error of the measurement method, 20 measurements were performed within half an hour. A fundamental power of $97.2 \% \pm 0.2 \%$ was determined (since the laser system had been optimized in the meantime, the value for the fundamental power differs from the one already presented). However, this statistical error includes the fluctuations of the modal composition of the laser system during the measurement time.

A consistency test was performed by measuring the light power that is reflected off the resonator when it is resonant to the fundamental mode. This can be easily calculated with the information of a Modescan in the case of an impedancematched resonator,

$$
P_{\text {refl }}(L)=P_{\text {tot }}-P(L) \text {. }
$$

The ratio $P_{\text {refl }}(L) / P_{\text {refl }}(L+\lambda / 2)$ was once calculated from a Modescan and was afterward measured with a photodiode. The calculated ratio was $2.5 \% \pm 0.2 \%$ and the measured value was $4.2 \% \pm 0.3 \%$. The difference of $1.7 \%$ cannot be

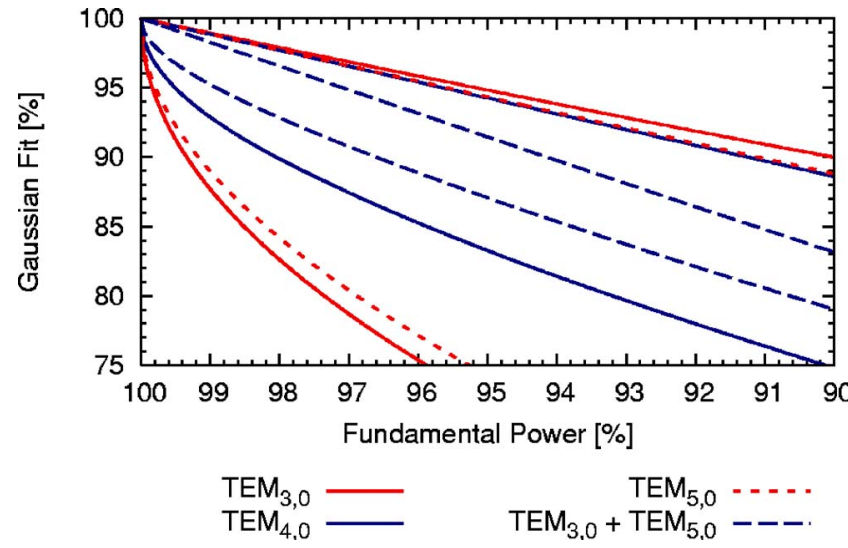

FIG. 5. (Color online) Numerical simulation of the dependence of the Gaussian fit value on the fundamental power for beams with different higher modes. For example, $95 \%$ fundamental power on the $x$ axis means that $5 \%$ of the power is in the higher mode(s) named in the legend. The $y$ axis shows then the value of the Gaussian fit. Two different curves are shown for each legend entry which correspond to two different phases between the higher mode(s) and the fundamental mode.

explained by statistical errors and must therefore originate from systematic errors. One error was made by using a quadrant photodiode to measure the ratio. Since the quadrant photodiode has a gap, the signal does not only depend on the power but also on the mode of the beam.

The overall power content of unidentified modes with an order greater than 24 was roughly estimated to be around $0.13 \%$. This number is based on a fit of the function $p(g)=a \exp (-d g)$ to the measurement data with $a$ and $d$ as free parameters (see Fig. 3), where the form of the fit function has no special physical motivation. Then the sum $\sum_{g=25}^{\infty} 2 p(g)$ was calculated.

For comparison, the $M^{2}$ factor was measured with a CCD camera. A factor $M^{2}$ of $1.12 \pm 0.04$ was determined, where the error is only due to statistical errors.

As can be seen from the numerical simulation (see Fig. 5), it is impossible to determine the fundamental power from the knowledge of only the Gaussian fit value. The Gaussian fit strongly depends on the mode order and even on the phase between the $\mathrm{TEM}_{00}$ mode and the higherorder mode. Therefore, the Gaussian fit is not suitable to quantify the beam quality for our purposes.

The calculation of the $M^{2}$ factor for different coherent beams (see Fig. 6) shows that the fundamental power cannot be determined from this factor. The $M^{2}$ factor depends as well, however, in a simpler way than in the case of the Gaussian fit, on the mode order and on the phase between higher modes.

In the end only the Modescan can be used from these three measurement methods to accurately determine the fundamental power. A simplified method can be used as well, where the power is measured that is reflected from the resonator locked to the $\mathrm{TEM}_{00}$ mode. In this case it is required that the resonator is impedance matched and one cannot gather further information about the mode composition of the beam compared to the Modescan. 


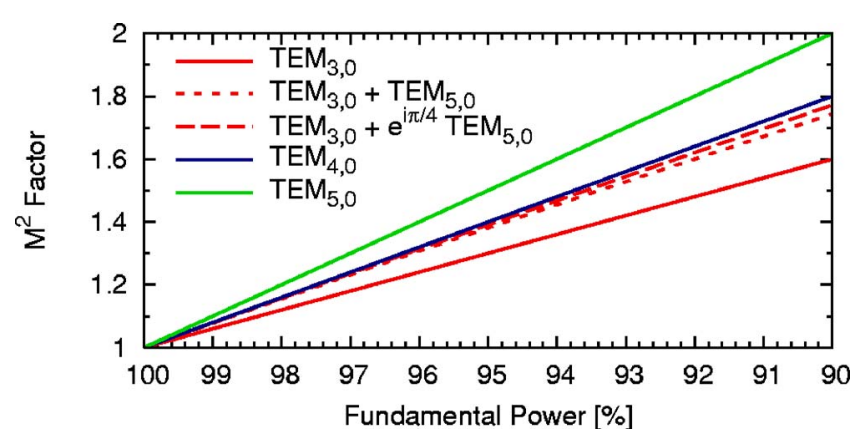

FIG. 6. (Color online) Dependence of the $M^{2}$ factor on the fundamental power for beams with different higher modes. The composition of the $\mathrm{TEM}_{30}$ and $\mathrm{TEM}_{50}$ modes was calculated once for zero phase angle between the fields and once with a phase angle of $45^{\circ}$.

\section{E. Discussion}

The measurement method presented here, the Modescan, is suited to characterize the beam quality and to determine the mode composition of laser beams. The fundamental power, which is the suitable quantity to measure the beam quality for many experiments, can be determined from the measurement data with small errors. Since the measurement duration is very short, this method lends itself to optimizing the beam quality of lasers, for example, by performing adjustments during the measurements.

The working principle has been confirmed in an experiment, and statistical and systematic errors were estimated. The statistical errors were within fractions of a percent and consistency checks with the reflected power of the resonator yielded an agreement within a few percent.

With our experiment it is possible to measure the fundamental power of single-frequency cw lasers at a wavelength of $1064 \mathrm{~nm}$. Since the only requirement for the laser is that it is a single-frequency cw laser, this method can be adapted to any other wavelength.

It is possible to replace the three-mirror ring resonator with a two-mirror linear resonator to avoid the ellipticity and the astigmatism of the fundamental mode, even though these deviations from an ideal $\mathrm{TEM}_{00}$ mode are negligible in our configuration. A linear resonator has the disadvantage that modes with even and odd $l$ cannot be distinguished and so less information about the beam is gathered.

In order to measure the fundamental power of a beam, the Modescan should be preferred to conventional $M^{2}$ factor measurements or Gaussian fit measurements, since the Modescan can deliver a substantially more accurate value.

\section{POINTING}

The pointing of a $\mathrm{TEM}_{00}$ beam can be expressed in first order by fluctuations of the $\mathrm{TEM}_{10}$ and $\mathrm{TEM}_{01}$ modes. ${ }^{22,25}$ If a $\mathrm{TEM}_{00}$ beam is shifted by $\delta x$ and tilted by $\delta \alpha$ in the $x$ direction, the complex amplitude $U(\delta x, \delta \alpha)$ of the beam can be expanded in Hermite-Gauss modes $\Phi_{l, m}$,

$$
U(\delta x, \delta \alpha) \approx \Phi_{0,0}+\epsilon \Phi_{1,0} \quad \text { with } \quad \epsilon=\frac{\delta x}{w_{0}}+i \frac{\delta \alpha}{\theta_{D}},
$$

where $\epsilon$ is a complex number that describes the beam position, $w_{0}$ is the radius of the beam waist, and $\theta_{D}$ is the half divergence angle of the $\mathrm{TEM}_{00}$ beam. The squared magnitude $|\epsilon|^{2}$ is the relative power of the $\mathrm{TEM}_{10}$ mode and is thus invariant under propagation through ideal optical systems.

This expansion corresponds to a transverse shift and angular tilt in the $x$ direction (horizontal). For a shift and tilt in the $y$ direction (vertical), the same expression is valid, if the $\mathrm{TEM}_{10}$ mode $\Phi_{1,0}$ is exchanged with the $\mathrm{TEM}_{01}$ mode $\Phi_{0,1}$.

The idea of our measurement method is to detect the complex quantity $\epsilon(t)$ by comparing the test beam with the fundamental mode of an optical resonator, which is used as a reference beam. The fundamental mode of a resonator can serve as a very stable beam pointing reference, as will be explained later (Sec. III A). The test beam, which impinges on the resonator, is superimposed at the input coupler of the resonator with the fundamental mode of the resonator. The fundamental mode is excited by the test beam itself. With the DWS the relative shift and tilt of the test beam in respect to the reference beam is measured. The DWS is explained in more detail in Ref. 17.

After the test beam is superimposed with the reference beam, the movement of optical components downstream toward the detection with quadrant photodiodes is irrelevant in first order. Therefore, the pointing of the test beam is measured at the superposition point.

The quantity $\epsilon(t)$ can be used to calculate different quantities that are used to describe the pointing of a laser beam. There is a direct relation between $\epsilon(t)$ and the pointing stability ${ }^{30} P_{s}$. The pointing stability is the time-averaged overlap $\left|\eta_{m}\right|^{2}$ between the test beam and the reference beam. If we use the expression from Eq. (19) as the complex amplitude for the test beam, we get

$$
\begin{aligned}
P_{s} & =\frac{1}{T} \int_{0}^{T}\left|\eta_{m}(t)\right|^{2} d t, \quad\left|\eta_{m}(t)\right|^{2} \\
& =\left|\frac{\int U_{\text {ref }}^{*} U(\delta x, \delta \alpha) d x d y}{\int\left|U_{\text {ref }}\right|^{2} d x d y}\right|^{2}=1-|\epsilon(t)|^{2} .
\end{aligned}
$$

The quantities $P_{s}$ and $\left|\eta_{m}\right|^{2}$ are, like $|\epsilon|^{2}$, invariant under propagation through ideal optical systems.

With the standard deviations $\sigma_{x}$ and $\sigma_{\alpha}$ of $\delta x$ and $\delta \alpha$, we can calculate the relative beam positional stability $\Delta x$ and the relative beam angular stability $\Delta \alpha$ which are defined in Ref. 13,

$$
\Delta x=\frac{4 \sigma_{x}}{2 w_{0}}, \quad \Delta \alpha=\frac{2 \sigma_{\alpha}}{2 \theta_{D}} .
$$

From the time series of $\epsilon$, we can thus calculate $P_{s}, \Delta x$, and $\Delta \alpha$ as well as the linear spectral density of $|\epsilon|$. This linear spectral density is often used in the field of interferometric gravitational wave detectors to characterize the pointing of a laser beam.

In addition to the DWS measurements, the quantity $|\epsilon|^{2}$ was measured directly by detecting the power fluctuations of the $\mathrm{TEM}_{10}$ and $\mathrm{TEM}_{01}$ modes. These power fluctuations normalized to the power of the $\mathrm{TEM}_{00}$ mode are equal to $|\epsilon|^{2}$ [see. Eq. (19)]. An optical resonator was used to separate the 
$\mathrm{TEM}_{10}$ and $\mathrm{TEM}_{01}$ modes from the remaining modes of the test beam. The power of the $\mathrm{TEM}_{10}$ and $\mathrm{TEM}_{01}$ modes were then measured with a photodiode.

\section{A. Setup}

An extended experimental setup (Fig. 1) compared to the Modescan setup was used in order to stabilize the resonator to the fundamental mode by using a dither lock and also to detect the DWS signals. The beam that is reflected off the resonator is split by a polarization independent beam splitter into two parts of equal power. These beams are detected by two quadrant photodiodes (QPDs). The QPDs were oriented in such a way that a set of top and bottom halves as well as left and right halves were available.

A lens system was calculated for the reflection path of the resonator, such that the beam widths at both QPDs are equal and that the Gouy phases of the $\mathrm{TEM}_{10}$ as well as of the $\mathrm{TEM}_{01}$ mode had a difference of $90^{\circ}$ between the locations of the two diodes. The beam width had a radius of $730 \mu \mathrm{m}$ and the difference of the Gouy phase between the superposition point at the input coupler of the resonator and the first QPD was $\eta_{1}=165^{\circ}$ and accordingly for the second QPD $\eta_{2}=\eta_{1}+90^{\circ}=255^{\circ}$. The QPDs had an active area with a diameter of $d=8 \mathrm{~mm}$ and were read out with transimpedance amplifiers.

The piezo at the curved mirror of the resonator was modulated with a small sinusoidal signal at $f_{d}=50 \mathrm{kHz}$ in order to generate phase modulation sidebands for the dither lock. The dither lock, which is described as transmission lock in Ref. 31, can be understood as a variant of the well known Pound-Drever-Hall lock $^{32,33}$ but with the phase modulation sidebands being generated in the resonator by length modulation.

The signals of the four quadrants of the QPDs were demodulated at the dithering frequency $f_{d}$. The bandwidth of the demodulated signal was restricted to $2 \mathrm{kHz}$ during this demodulation. The sum of the demodulated quadrant signals of one QPD was used as an error signal for stabilizing the resonator to the test beam. Therefore, the resonance frequency of the $\mathrm{TEM}_{00}$ mode was controlled by changing the resonator length with the piezo to match the frequency of the test beam.

The differences of the demodulated signals of the left and right halves $\left(d x_{1 / 2}\right)$ and the top and bottom halves $\left(d y_{1 / 2}\right)$ were calculated by analog electronics. These signals are, according to the DWS, proportional to $\Im\left(\epsilon e^{i \eta_{1 / 2}}\right)$. By measuring the signal of two QPDs with $\left|\eta_{1}-\eta_{2}\right|=90^{\circ}$, the quantity $\epsilon$ can be reconstructed.

Two mirrors in front of the resonator, which could be electronically tilted by piezo elements, were used to calibrate the DWS signals. The dependence of the tilt angle from the electronic signal was known and was used as a reference for the calibration.

The complete setup, including the laser system, was mounted on a vibration isolated optical table. The fundamental mode of the resonator is used as a reference beam and is defined by the positions of the three mirrors of the resonator. The mirrors were glued on an Invar spacer, which in turn was mounted on a rigid aluminum mount, which then was mounted on the breadboard.

Since the light field in the resonator is driven by the test beam itself, the pointing of the reference beam that leaves the resonator is also wiggling. This fluctuation of the pointing is, however, strongly suppressed by the mode-cleaning feature of the resonator and can therefore be neglected as shown in the following: Assume that a beam with a complex amplitude given by Eq. (19) is coupled into the resonator and that the resonator is locked to the $\mathrm{TEM}_{00}$ mode. Since the field $\Phi_{1,0}$ is then not resonant, it will be suppressed by a factor $G$ in the corresponding field that leaves the resonator, with

$$
G=\frac{1-r}{1-r e^{-i \phi}} .
$$

This factor depends on the reduction factor $r$ for the field amplitude for one round trip in the resonator and on the resonance phase $\phi$ of the $\mathrm{TEM}_{10}$ mode and $\mathrm{TEM}_{01}$ mode, respectively. The beam that leaves the resonator is now superimposed with the reflected part of the test beam. This yields for an impedance-matched resonator,

$$
U_{\text {refl }} \propto \Phi_{0,0}+\epsilon \Phi_{1,0}-\left(\Phi_{0,0}+\epsilon G \Phi_{1,0}\right)=\epsilon(1-G) \Phi_{0,1} .
$$

The pointing fluctuations of the beam that leaves the resonator and is used as reference beam are suppressed by $|G|$. For the resonator that is used for our measurements with $\mathcal{F}=200$ and a resonance phase of $\phi_{x}=0.95+\pi$ and $\phi_{y}=0.95$, respectively, the factors $G_{x}=8.8 \times 10^{-3} e^{-0.47 i}$ and $G_{y}=1.7 \times 10^{-2} e^{1.1 i}$, respectively, are evaluated.

The magnitude $|1-G|$ is taken into account in the calibration procedure which is described in Sec. III B. The phase rotation of $\epsilon$ which is introduced by $1-G$ is neglected. This phase rotation is $0.2^{\circ}$ for $\epsilon_{x}$ and $0.9^{\circ}$ for $\epsilon_{y}$.

\section{B. Measurement and analysis}

The test beam was coupled into the resonator and the alignment as well as the mode matching were optimized as already described in the context of the Modescan. The resonator was locked with a feedback loop to the fundamental mode with a bandwidth of $1.6 \mathrm{kHz}$.

The demodulated difference signals $\left(d x_{1}, d x_{2}, d y_{1}\right.$, and $d y_{2}$ ) were sampled with a 16 bit analog/digital (A/D) converter card at a sampling frequency of $20 \mathrm{kHz}$ for $60 \mathrm{~s}$. With these, the time series $\epsilon(t)$ was calculated,

$$
\boldsymbol{\epsilon}_{x} \propto\left(d x_{1}+i d x_{2}\right) e^{-i \eta_{1}}, \quad \epsilon_{y} \propto\left(d y_{1}+i d y_{2}\right) e^{-i \eta_{1}} .
$$

To eliminate laser power fluctuations the laser power was simultaneously sampled with another photodiode (not shown in Fig. 1) in front of the resonator.

In order to calibrate the measurement, a calibration signal was added to the piezo mirrors. The expected $\epsilon$ signal was calculated from the position of the piezo mirror relative to the resonator and compared with the actual measurement.

To verify the measurement method and its calibration, the beam pointing was further measured in a different way. For these purposes the resonator was locked to the $\mathrm{TEM}_{10}$ mode by introducing a small misalignment in the $x$ direction. 


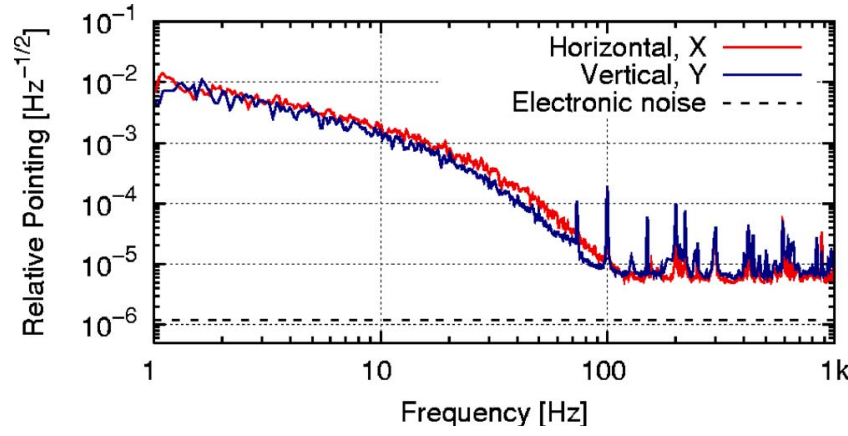

FIG. 7. (Color online) Linear spectral density of relative pointing $|\epsilon|$ in the $x$ and $y$ directions.

Again the dither lock was used to stabilize the resonator to this mode with a bandwidth of $1.6 \mathrm{kHz}$. If a complex amplitude given by Eq. (19) is coupled into such a stabilized resonator, then the following field will be transmitted:

$$
U_{\text {trans }} \propto G \Phi_{0,0}+\epsilon \Phi_{1,0} .
$$

Now the fundamental mode $\mathrm{TEM}_{00}$ is suppressed by the factor $G$, which was already calculated for our setup. The power of the transmitted beam essentially consists of the power of the $\mathrm{TEM}_{10}$ mode which is proportional to $|\epsilon|^{2}$. The part of the transmitted power of the $\mathrm{TEM}_{00}$ mode is only a constant offset.

The transmitted power was measured with a photodiode and sampled with the A/D card. With another photodiode the power of the test beam was sampled to take its power fluctuations into account. Afterward, the resonator was locked to the $\mathrm{TEM}_{00}$ mode to measure the power of the transmitted $\mathrm{TEM}_{00}$ mode power to determine the relative transmitted power in the $\mathrm{TEM}_{10}$ mode which is used to calculate $|\epsilon|^{2}$.

Two measurements are necessary in order to measure the pointing in the $x$ and $y$ directions. This is due to the fact that the resonance frequencies of the $\mathrm{TEM}_{10}$ and $\mathrm{TEM}_{01}$ modes differ.

\section{Results}

The measurements that are presented here were obtained with a Nd:YAG solid state ring laser (Innolight Mephisto), a so-called nonplanar ring oscillator (NPRO) ${ }^{34}$ The output power was $800 \mathrm{~mW}$ at a wavelength of $1064 \mathrm{~nm}$. The power of the $\mathrm{TEM}_{00}$ mode was $>96 \%$ and the laser oscillated only

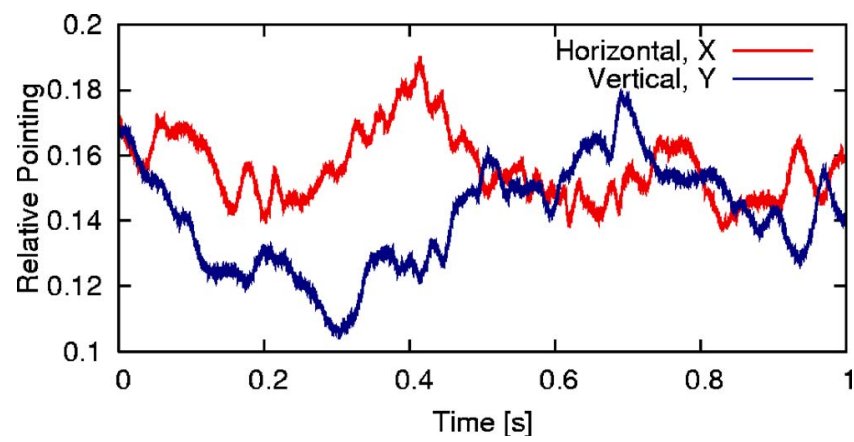

FIG. 8. (Color online) Time series of the relative pointing $|\epsilon|$ in the $x$ and $y$ directions. [see Eq. (19) for the definition of $\epsilon$ ].
TABLE I. Quantities calculated from the complex $\epsilon$ time series in a frequency band from $1 \mathrm{~Hz}$ to $1 \mathrm{kHz}$.

\begin{tabular}{lll}
\hline \hline & Horizontal & Vertical \\
\hline Standard deviation & $\left|\epsilon_{x}\right|=18 \times 10^{-3}$ & $\left|\epsilon_{y}\right|=15 \times 10^{-3}$ \\
Pointing stability & $P_{s}=1-324 \times 10^{-6}$ & $P_{s}=1-225 \times 10^{-6}$ \\
Relative beam positional stability & $\Delta x=31 \times 10^{-3}$ & $\Delta y=31 \times 10^{-3}$ \\
Relative beam angular stability & $\Delta \alpha_{x}=11 \times 10^{-3}$ & $\Delta \alpha_{y}=11 \times 10^{-3}$ \\
\hline \hline
\end{tabular}

in one longitudinal mode. About $200-250 \mathrm{~mW}$ was splitted from the main beam to measure the beam pointing.

The linear spectral density of $|\epsilon|$ (see Fig. 7) for the $x$ and $y$ directions differs only barely. Below $100 \mathrm{~Hz}$ the pointing fluctuations are increasing. A hypothesis, namely, that the pointing between 1 and $100 \mathrm{~Hz}$ is produced by acoustic noise from a flow box, was ruled out. Above $100 \mathrm{~Hz}$ many sharp peaks can be identified in the spectrum. We assume that among harmonics of the mains frequency of $50 \mathrm{~Hz}$, these peaks are produced by mechanical resonances of the optical components.

The electronic noise of the measurement was equivalent to approximately $|\epsilon|=1 \times 10^{-6} / \sqrt{\mathrm{Hz}}$. It was measured with dark QPDs. This corresponds to a pure angular tilt sensitivity of $1 \mathrm{nrad} / \sqrt{\mathrm{Hz}}$ or a pure transverse shift sensitivity of $0.4 \mathrm{~nm} / \sqrt{\mathrm{Hz}}$. The fundamental sensitivity limit is given by the shot noise of the light at the QPDs. ${ }^{17}$ This fundamental limit was, however, far below the electronic noise limit.

A part of the time series of $|\epsilon|$ is shown in Fig. 8. Several statistical quantities were calculated from the time series of $\epsilon$ for a frequency band from $1 \mathrm{~Hz}$ to $1 \mathrm{kHz}$ (see Table I). The measurements of $\epsilon$ using the DWS method were compared with the measurements of $|\epsilon|$, where the transmitted powers of the $\mathrm{TEM}_{10}$ and $\mathrm{TEM}_{01}$ modes were detected. The comparison of the linear spectral density of $|\epsilon|$ shows a good agreement (see Fig. 9). The calibration of the DWS signals was hereby verified.

\section{Discussion}

The measurement of pointing fluctuations with the method presented here has been successfully demonstrated on a laser system. A good agreement with an alternative measurement method was achieved. The sensitivity was approximately $|\epsilon|=1 \times 10^{-6} / \sqrt{\mathrm{Hz}}$.

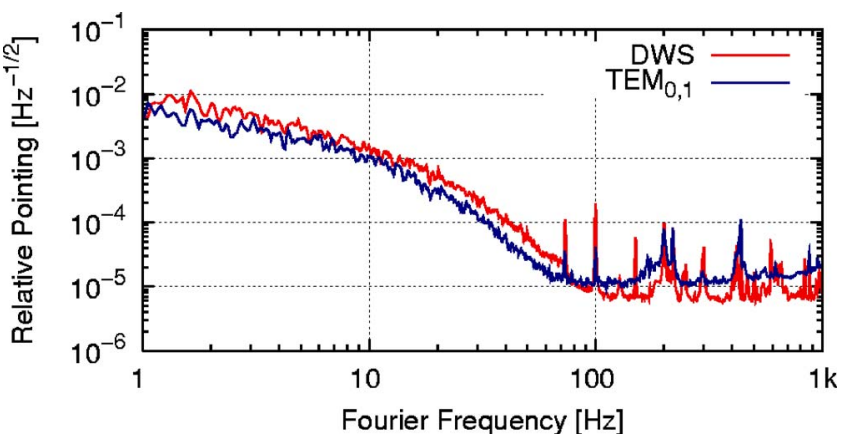

FIG. 9. (Color online) Comparison of the linear spectral densities of the relative pointing $|\epsilon|$ in the $y$ direction for two different measurement methods. 
The advantage of this measurement method is that a real laser beam is used as reference and therefore the transverse shift as well as the angular tilt can be measured at one point along the beam axis. Compared with conventional methods, which need, for example, two PSDs, those measurement errors that are caused by a relative movement of the PSDs are avoided.

This measurement method can be offhand transferred to any single-frequency cw laser that has a high fraction of its power in the $\mathrm{TEM}_{00}$ mode. In principle, there is no problem to measure the pointing fluctuations below $1 \mathrm{~Hz}$ or above $1 \mathrm{kHz}$.

This compact diagnostic unit is used as a tool for developing lasers for interferometric gravitational wave detectors, where a quick and reliable measurement of the beam quality is necessary to optimize the laser. The methods described will be used to monitor the short and long term performance of such laser systems in operation.

\section{ACKNOWLEDGMENTS}

The authors would like to thank Bastian Schulz, Maik Frede, and the Laser Zentrum Hannover for providing the $\mathrm{Nd}: \mathrm{YVO}_{4}$ amplifier and Albrecht Rüdiger for his helpful comments during the preparation of this article.

${ }^{1}$ A. E. Siegman, Proc. SPIE 1224, 2 (1990).

${ }^{2}$ ISO-11146 lasers and laser-related equipment-test methods for laser beam parameters-beam widths, divergence angle, and beam propagation factor, 1999.

${ }^{3}$ B. Willke, N. Uehara, E. Gustafson, R. L. Byer, P. J. King, S. U. Seel, and R. L. Savage, Opt. Lett. 23, 1704 (1998).

${ }^{4}$ P. Weßels and C. Fallnich, Opt. Express 11, 3346 (2003).

${ }^{5}$ E. Tervonen, J. Turunen, and A. T. Friberg, Appl. Phys. B: Photophys. Laser Chem. 49, 409 (1989).

${ }^{6}$ A. E. Siegman and S. W. Townsend, IEEE J. Quantum Electron. 29, 1212 (1993).
${ }^{7}$ A. Cutolo, T. Isernia, I. Izzo, R. Pierri, and L. Zeni, Appl. Opt. 34, 7974 (1995).

${ }^{8}$ A. Liesenhoff and F. Rühl, Rev. Sci. Instrum. 66, 4059 (1995).

${ }^{9}$ F. Gori, M. Santarsiero, R. Borghi, and G. Guattari, Opt. Lett. 23, 989 (1998).

${ }^{10}$ M. A. Golub, A. M. Prokhorov, I. N. Sisakyan, and V. A. Soifer, Sov. J. Quantum Electron. 12, 1208 (1982).

${ }^{11}$ Z. Y. Wang, T. Chen, P. He, and T. C. Zuo, Opt. Commun. 175, 215 (2000).

${ }^{12}$ M. R. Sayeh, H. R. Bilger, and T. Habib, Appl. Opt. 24, 3756 (1985).

${ }^{13}$ ISO-11670 lasers and laser-related equipment-test methods for laser beam parameters-beam positional stability, 2003.

${ }^{14}$ M. Levesque, A. Mailloux, M. Morin, P. Galarneau, Y. Champagne, O. Plomteux, and M. Tiedtke, Proc. SPIE 2870, 216 (1996).

${ }^{15}$ R. Maestle, W. Plass, J. Chen, Chr. Hembd-Soellner, A. Giesen, H. Tiziani, and H. Hugel, Proc. SPIE 2870, 319 (1996).

${ }^{16}$ S. Grafström, U. Harbarth, J. Kowalski, R. Neumann, and S. Noehte, Opt. Commun. 65, 121 (1988).

${ }^{17}$ E. Morrison, B. J. Meers, D. I. Robertson, and H. Ward, Appl. Opt. 33, 5041 (1994).

${ }^{18}$ H. Grote et al., Appl. Opt. 43, 1938 (2004).

${ }^{19}$ A. Romann, D. S. Rabeling, G. Vine, J. H. Chow, M. B. Gray, and D. E. McClelland, Gen. Relativ. Gravit. 37, 1591 (2005).

${ }^{20}$ N. M. Sampas and D. Z. Anderson, Appl. Opt. 29, 394 (1990).

${ }^{21}$ G. Müller, DCC Report No. LIGO-T020022-01-D, 2003 (unpublished).

${ }^{22}$ A. Rüdiger, R. Schilling, L. Schnupp, W. Winkler, H. Billing, and K. Maischberger, Opt. Acta 28, 641 (1981).

${ }^{23}$ A. E. Siegman, Lasers (University Science Books, Mill Valley, CA, 1986).

${ }^{24}$ N. Uehara, E. K. Gustafson, M. M. Fejer, and R. L. Byer, Proc. SPIE 2989, 57 (1997).

${ }^{25}$ D. Z. Anderson, Appl. Opt. 23, 2944 (1984).

${ }^{26}$ I. Kimel and L. R. Elias, Int. J. Quantum Chem. 29, 2562 (1993).

${ }^{27}$ DataRay Inc., Application note: Beam fit algorithms, 2004.

${ }^{28}$ M. Hildebrandt, M. Frede, P. Kwee, B. Willke, and D. Kracht, Opt. Express 14, 11071 (2006).

${ }^{29}$ I. Zawischa et al., Class. Quantum Grav. 19, 1775 (2002).

${ }^{30}$ M. Morin, P. Bernard, and P. Galarneau, Opt. Lett. 19, 1379 (1994).

${ }^{31}$ A. D. White, Int. J. Quantum Chem. 1, 349 (1965).

${ }^{32}$ E. D. Black, Am. J. Phys. 69, 79 (2001).

${ }^{33}$ R. W. P. Drever, J. L. Hall, F. V. Kowalski, J. Hough, G. M. Ford, A. J. Munley, and H. Ward, Appl. Phys. B: Photophys. Laser Chem. 31, 97 (1983).

${ }^{34}$ I. Freitag, A. Tünnermann, and H. Welling, Proc. SPIE 2379, 335 (1995). 